Kardiologe 2019 $13: 330$

https://doi.org/10.1007/s12181-019-00352-5

Online publiziert: 10. Oktober 2019

(c) Springer Medizin Verlag GmbH, ein Teil von Springer Nature 2019

\section{Originalpublikation}

Mehta SR, Wood DA, Storey RF et al (2019) Complete revascularization with multivessel PCl for myocardial infarction. New Engl J Med. https://doi.org/10.1056/NEJMoa1907775 (epub ahead of print)

Beim ST-Hebungsinfarkt stellt sich die Frage, ob neben der Infarktläsion („,culprit lesion“) auch begleitende höhergradige Stenosen interveniert werden sollen, um das Risiko für weitere kardiovaskuläre Ereignisse zu reduzieren.

\section{Zusammenfassung der Studie}

Die COMPLETE-Studie ist dieser Frage nachgegangen und hat 4041 Patienten mit erfolgreicher PCI der „culprit lesion“ weiter in 2 Gruppen randomisiert:

- 1. komplette Revaskularisation mit

PCI aller angiographisch relevanter

Stenosen (stratifiziert für im gleichen

Aufenthalt oder im Verlauf) und

- 2. Verzicht auf weitere geplante PCI.

Die 2 primären Endpunkte waren definiert als kardiovaskulärer Tod oder Myokardinfarkt bzw. kardiovaskulärer Tod, Myokardinfarkt oder Ischämie-getriebene Revaskularisation. Nach einem Follow-up von im Median 3 Jahren zeigte die Gruppe mit kompletter Revaskularisation signifikant weniger Endpunkte als die „culprit lesion only“ Gruppe (7,8 vs. $10,5 \%$ bzw. 8,9 vs. $16,7 \%)$. Interessanterweise war der Vorteil für die komplette Revaskularisationsstrategie unabhängig davon, ob im gleichen Aufenthalt

Nikos Werner

Herzzentrum Trier, Innere Medizin III, Krankenhaus der Barmherzigen Brüder, Trier, Deutschland

\title{
Fokus auf die Infarktläsion beim ST-Hebungsinfarkt (STEMI) - die übrigen Stenosen im Intervall versorgen!
}

(Median 1 Tag nach Randomisierung) oder im Intervall (Median 23, Interquartilbereich 12,5-33,5 Tage) die übrigen Stenosen interveniert wurden.

\section{Kommentar}

Die COMPLETE-Studie hat große Relevanz für die Klinik: Im STEMI sollte die Infarktläsion unser klarer Fokus sein. Umgekehrt benötigen wir eine sorgfältige angiographische Dokumentation des übrigen Koronarsystems, um begleitende „non culprit“ Stenosen zu identifizieren. Die in der Studie beschriebenen „non culprit“ Läsionen lagen angiographisch im Bereich 78-79\% Stenosegrad und betrafen in ca. $40 \%$ der Fälle den Ramus interventricularis anterior. Eine medikamentöse Therapie dieser Stenosen war in COMPLETE nicht ausreichend. Nur die komplette Revaskularisation auch der „non culprit“ Läsionen (90,1 \% der Patienten hatten nach PCI SYNTAX-Score 0) war mit einer Reduktion der kombinierten Endpunkte assoziiert. Hier waren vor allem die Raten an Myokardinfarkten, ischämiegetriebenen Revaskularisationen und instabiler Angina die treibenden Ereignisse zuungunsten der „culprit only“ Gruppe. Blutungskomplikationen und kontrastmittelinduziertes Nierenversagen waren nicht unterschiedlich zwischen den Gruppen. Die Cross-overRate innerhalb der ersten 45 Tage war in beiden Gruppen niedrig: 3,9\% der Patienten wechselten von der kompletten Revaskularisationsgruppe in die rein konservativen Gruppe, umgekehrt wechselten $4,7 \%$ in die komplette Re- vaskularisationsgruppe. Nachteil des Studiendesigns war die niedrige $\mathrm{Ra}$ te an FFR(fraktionierte Flussreserve)Messungen. Diese erfolgte nur im Bereich eines Stenosegrads von 50-69\%, während die überwiegende Zahl an Stenosen rein angiographisch eingeschätzt und interveniert wurden.

Zusammenfassend zeigt die COMPLETE-Studie, dass die weitere Versorgung von angiographisch hochgradig imponierenden Stenosen innerhalb von ca. 30 Tagen nach Infarkt wichtig ist und im weiteren Verlauf zu einer Reduktion ischämischer Endpunkte führt, ohne die Sicherheit des Patienten zu gefährden.

\section{Korrespondenzadresse}

\section{Prof. Dr. Nikos Werner}

Herzzentrum Trier, Innere Medizin III, Krankenhaus der Barmherzigen Brüder Nordallee 1, 54292 Trier, Deutschland n.werner@bk-trier.de

Interessenkonflikt. N. Werner hat Vortragshonorare der Firmen Abbott, Boston Scientific, Edwards Lifesciences und Medtronic erhalten. NW ist Proktor für Medtronic. 